\title{
Reaction Network Modeling of Complex Ecological Interactions: Endosymbiosis and Multilevel Regulation
}

\author{
Tomas Veloz iD $^{1,2,3}$ and Daniela Flores $\mathbb{D}^{1,4}$ \\ ${ }^{1}$ Fundación para el Desarrollo Interdisciplinario de la Ciencia, la Tecnología y Las Artes, Arturo Prat 249, Santiago, Chile \\ ${ }^{2}$ Universidad Andres Bello, Departamento Ciencias Biologicas, Facultad Ciencias de la Vida, Santiago 8370146, Chile \\ ${ }^{3}$ Centre Leo Apostel, Vrije Universiteit Brussel, Rue de la Strategie 33, 1060, Brussels, Belgium \\ ${ }^{4}$ Universidad de Chile, Departamento de Biología, Facultad de Ciencias, Nuñoa, Santiago, Chile \\ Correspondence should be addressed to Tomas Veloz; tveloz@gmail.com
}

Received 10 July 2020; Accepted 29 July 2021; Published 9 August 2021

Academic Editor: Abdellatif Ben Makhlouf

Copyright (c) 2021 Tomas Veloz and Daniela Flores. This is an open access article distributed under the Creative Commons Attribution License, which permits unrestricted use, distribution, and reproduction in any medium, provided the original work is properly cited.

\begin{abstract}
Endosymbiosis is a type of symbiosis where one species of microscopic scale inhabits the cell of another species of a larger scale, such that the exchange of metabolic byproducts produces mutual benefit. These benefits can occur at different biological levels. For example, endosymbiosis promotes efficiency of the cell metabolism, cell replication, and the generation of a macroscopic layer that protects the organism from its predators. Therefore, modeling endosymbiosis requires a complex-systems and multilevel approach. We propose a model of endosymbiosis based on reaction networks, where species of the reaction network represent either ecological species, resources, or conditions for the ecological interactions to happen, and the endosymbiotic interaction mechanisms are represented by different sequences of reactions (processes) in the reaction network. As an example, we develop a toy model of the coral endosymbiotic interaction. The model considers two reaction networks, representing biochemical traffic and cellular proliferation levels, respectively. In addition, the model incorporates top-down and bottom-up regulation mechanisms that stabilizes the endosymbiotic interaction.
\end{abstract}

\section{Introduction}

Endosymbiosis is a particular kind of symbiosis which occurs when an organism (endosymbiont) lives within another (host) in a mutualistic relationship. This relationship is given by the coupling of the host's and endosymbiont's metabolisms through exchange of useful metabolites $[1,2]$. The endosymbiotic concept was proposed by Lynn Margulis, who developed Serial Endosymbiosis Theory (SET) to explain the origin of plastids and mitochondria as organelles in the eukaryotic cell, promoting the emergence of eukaryotic cell complexity [3]. Endosymbiosis is recognized as a widespread mechanism in nature and is taking relevance as an evolutionary mechanism in different lineages [4]. Symbiotic interactions allow alternative ways of evolution, for example, through natural selection, in evolving complexity for a population of individuals. Endosymbiosis implies the coupling of two or more species in an intimate relationship.
Such relation leads to an increase of phenotypic complexity at the genomic, physiological, and morphological levels. Therefore, endosymbiosis enables ecological expansion into different and new niches. As Darwin said in his book The Origin of Species: "Natural Selection cannot possibly produce any modification in a species exclusively for the good of another species; although throughout nature one species incessantly takes advantage of, and profits by, the structures of other" [5].

One of the examples of endosymbiosis corresponds to the relationship between corals (Cnidaria, Anthozoa) and a photosynthetic algae of the genus Symbiodinium or Zooxanthellae [6], in which the latter lives within the gastrodermis coral cells. This relationship is typical when the environmental food is scarce $[7,8]$, and they (the Symbiodinium and the coral) can feed back through their metabolisms, by the nutrient acquisition via the cycling of organic compounds which supplies extra energy to both 
endosymbiont and host. The latter provides an ecological advantage and thus promotes survival because the metabolic coupling between coral cells and Symbiodinium promotes cellular proliferation implying coral's growth. Interestingly, such growth process is regulated by the release of an inhibition factors by the endosymbiont. Namely, when certain byproducts of the energy generation process that might harm the cells surpass a threshold, inhibition factors are released, stopping the growth process $[9,10]$. Thus, endosymbiosis is a multilevel and self-regulated process.

The establishment of endosymbiosis gives rise to new structural and morphological properties and thus to new evolutionary competences. However, the experimental study of endosymbiotic systems is extremely complicated, and it cannot be properly achieved by analyzing the interacting species separately [1]. For this reason, a modeling framework for the establishment of the endosymbiosis relationship is of crucial importance from an ecological perspective [11].

Such modeling framework of endosymbiosis should incorporate both the multiple levels at which the interaction takes place as well as bottom-up and top-down regulation mechanisms. Interestingly, these features are relevant not only for endosymbiosis but also for embryonic development [12], ecological trophic networks [13], and also during evolutionary processes $[14,15]$. For this reason we aim at proposing a process-based and multilevel approach to the representation of endosymbiosis.

Reaction networks, and equivalent languages such as Petri Nets [16], are the main representational languages in systems biology $[17,18]$. They allow for a description of the dynamical properties of complex biochemical reaction system of multilevel nature, using methods that exploit both the structure and stoichiometry of the network $[19,20]$. Reaction networks can also describe processes whose entities are not only biochemical [21]. Indeed, reaction networks have been applied to model the exchange of economic goods [22], the influence of political decisions [23], the evolution of cooperation [24], and other game-theoretical settings [25]. Therefore, reaction networks can not only be applied outside biochemical settings, but also have the potential to become an intuitive language to describe and study problems of multidisciplinary nature from a systemic perspective $[26,27]$. The latter can have novel and powerful applications particularly in the modeling of ecological interactions [28].

In the present study, we provide a simple mathematical model of the interaction between anthozoan corals and symbiont algae of the genus Symbiodinium by representing the mechanisms of their endosymbiotic interaction using reaction networks. In particular, we propose two reaction networks modeling endosymbiosis at a biochemical traffic (lower level) and cellular proliferation (upper level). We point to how endosymbiosis occurs at each level and focus on representing the multilevel regulatory mechanisms that are known to happen in this two-layer interaction process.

The paper is organized as follows. In Section 2, we review in detail the endosymbiotic mechanisms known up to date. In Section 3, we introduce the basics of ecological modeling using reaction networks. In Section 4, we propose a toy model of the endosymbiotic as well as of the multilevel regulatory mechanisms of the Symbiodinium. We complement the latter with a discussion of how we could scale up to more realistic models of the interaction, and we end with a conclusion.

\section{Endosymbiotic Mechanisms and Their Levels of Interaction}

Corals are ancestral animals formed by only four epithelial layers: oral ectoderm, oral endoderm, aboral endoderm, and calcidodermis (Figure 1). In conditions of food limitations, corals (Cnidaria) can engulf photosynthetic dinoflagellates (algae) of the genus Symbiodinium from the surrounding water. Symbiodinium cells engulfed by the coral are hosted gastrodermis layer of the coral that surrounds the gastric cavity (oral endoderm), where they can still perform photosynthesis. Namely, the Symbiodinium acquires nutrients and metabolic residual compounds from the host and produces organic compounds. These organic compounds that are product of the photosynthesis not only foster the growth and respiration of endosymbionts, but also are used to produce nearly up to $95 \%$ of the host's energy demand [29].

The latter phenomenon occurs in the oral endoderm (yellow layer) within a membrane called symbiosome (white layer), produced by both the host and the Symbiodinium (green layer) in Figure 1.

The endosymbiotic interaction occurs in various phases: (1) initial contact; (2) engulfment; (3) sorting; (4) proliferation; (5) dynamic stability; and (6) dysfunction (Figure 2). The initial contact phase is characterized by the compatibility recognition between the host and the symbiont (Figure 2(a)). Namely, each coral lineage is compatible with one or more Symbiodinium lineages [31]. When compatible, the coral engulfs the algae (Figure 2(b)). After successful engulfment, the symbiosome membrane is developed promoting the stabilization and persistence of the symbiont within the cells of the coral [32]. Next, the persistence of the symbiont depends on the balance between host cell growth and proliferation of the symbiont population. The latter balance can be achieved by a series of sorting and proliferation steps, reaching the dynamic stability phase (Figure 2(c)). This last stage is characterized by the metabolic exchange and nutrient trafficking, where the products generated by dinoflagellate support coral metabolism, growth, reproduction, and survival and also promote the conservation and recycling of essential nutrients. In this phase, the typical calcium deposition of coral reef takes place. This phenomenon is strongly promoted by the endosymbiosis [33]. This calcium matrix deposition protects the coral against predators and decreases mechanical erosion of the marine environment, further promoting its persistence and growth [34] (Figure 2). The last phase of the endosymbiotic interaction is dysfunction, related to the bleaching of the calcium layer, which is explained by the Symbiodinium expulsion by exocytosis and coral mortality, and by host cell detachment or host cell apoptosis, generally due by the production of reactive oxygen species or cellular signaling [35]. Coral reef bleaching is increasingly 


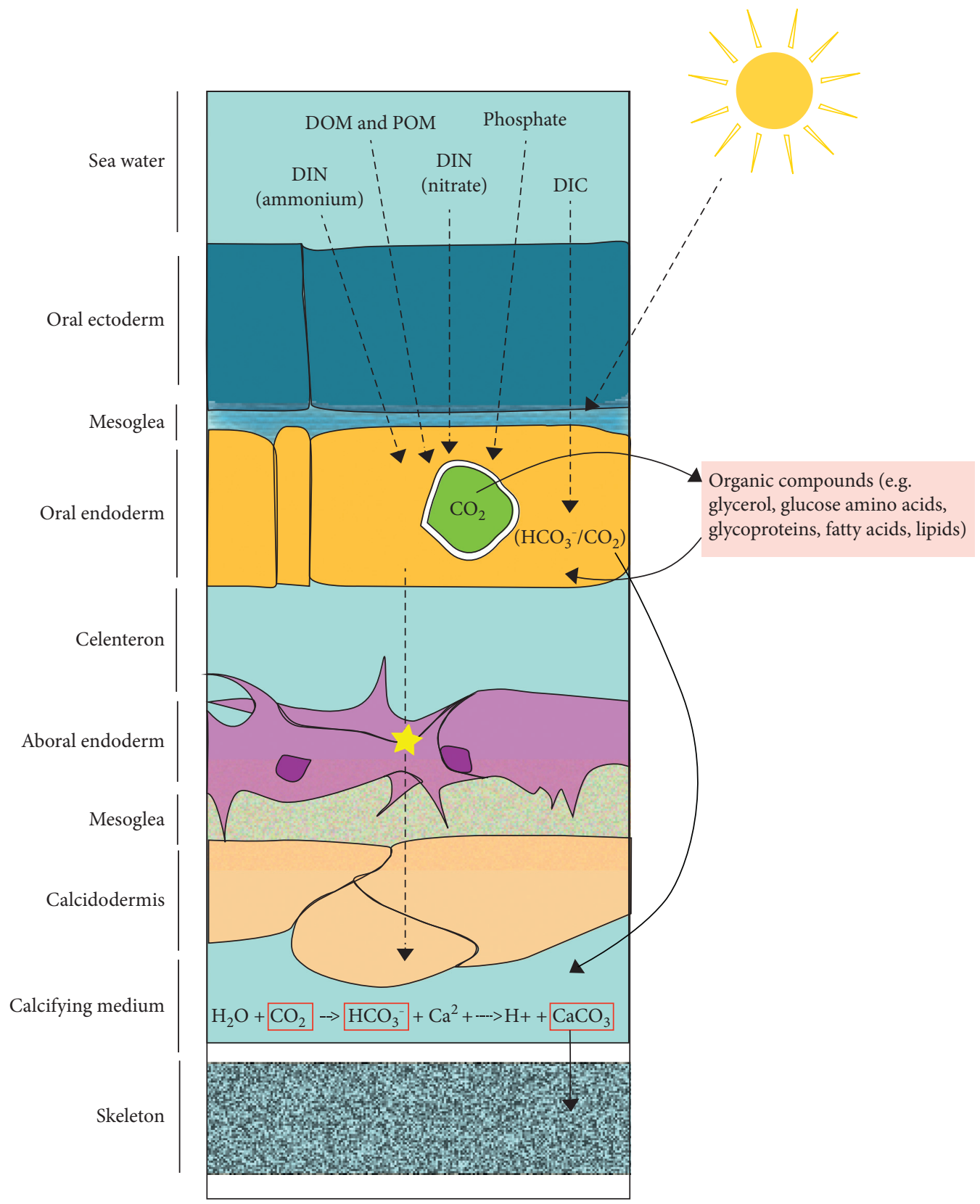

FIGURE 1: Metabolic interactions and calcification processes. DIC: dissolved inorganic carbon uptake; DOM: dissolved organic matter; POM: particulate organic matter; DIN: dissolved inorganic nitrogen.

threatened by recent changes in salinity, higher solar radiation, increased sedimentation or pollutants, elevated sea surface temperatures, and global warming [36].

In the dynamic stability phase, coral and algae metabolisms couple controlling each other's growth, promoting functional efficiency $[37,38]$. Typically, coral gastrodermis regulate their density to maintain up to two Symbiodinium per cell $[39,40]$. Such regulation incorporates various mechanisms, such as the expulsion and degradation of Symbiodinium, signaling factors affecting the cellular cycle such as a host release factors (HRFs) $[9,10]$, and other processes and biochemical factors that act as inhibitory factors (IFs) released by the host, hindering the growth of symbionts [41-43]. The release of IFs depends on the concentration of photosynthetic byproducts in the metabolism of the endosymbiont. Thus, this factor is relevant to achieve a steady growth and nutrient traffic state.

In steady state, Symbiodinium is benefited by acquisition of inorganic carbon, inorganic nitrogen, and inorganic phosphate by the coral, which gives the Symbiodinium greater availability of these nutrients for its own growth. In turn, as a product of the photosynthesis of Symbiodinium, diverse compounds are generated that nourish the coral, such as glycerol, amino acids, glucose, maltose, Krebs cycle molecules, pyruvate, zooxanthellamine, and zooxanthellatoxins [44]. In this way, the coral develops mechanisms and processes that 


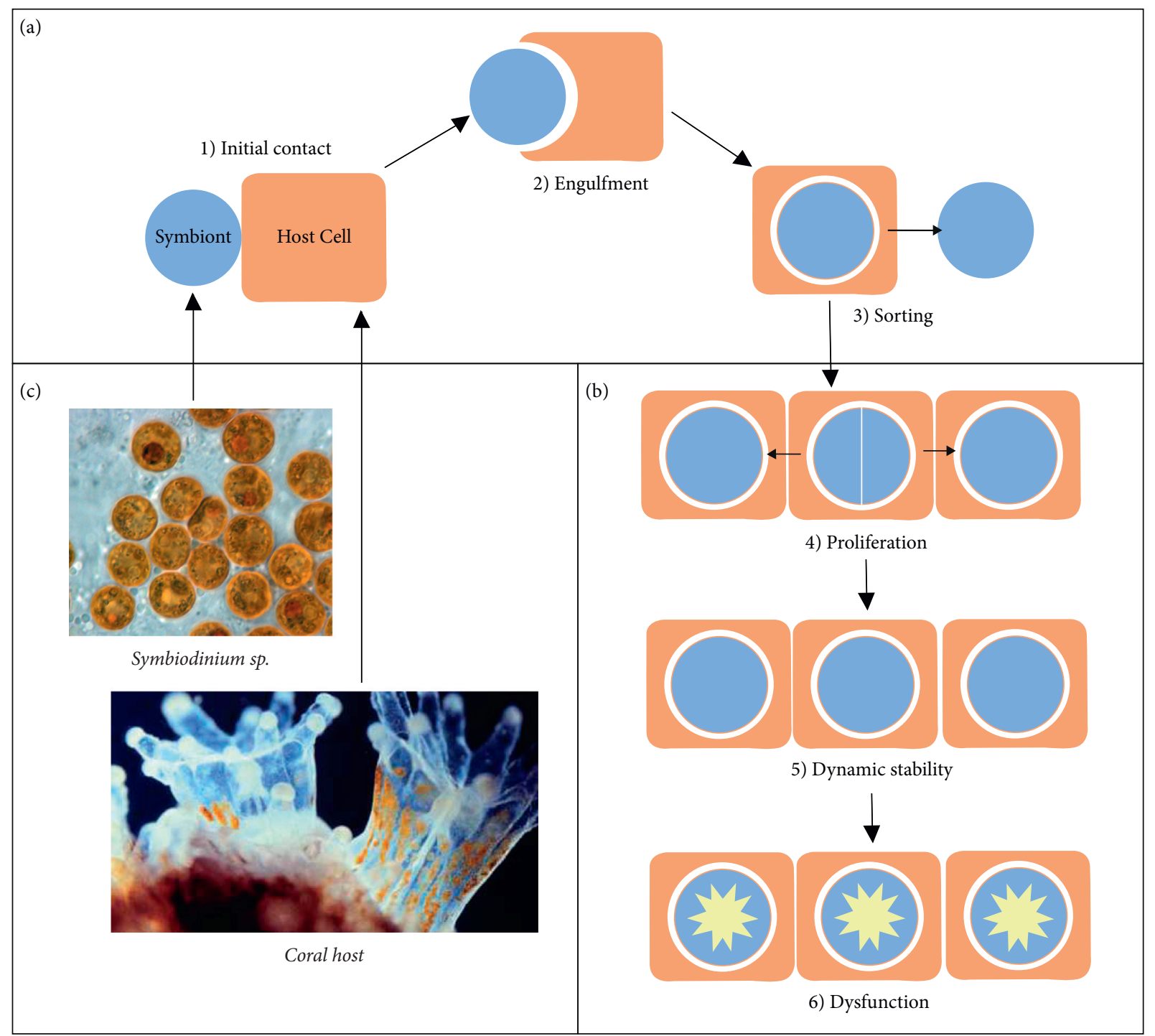

FIGURE 2: Phases of endosymbiosis establishment and persistence in cnidarian-Symbiodinium interaction (modified from [30]).

benefit endosymbiont and vice versa. These mechanisms can affect other processes occurring at other cellular layers of the host. For example, in the carbon metabolism, coral actively transports carbon from outside to gastrodermis cells and concentrates $\mathrm{CO}_{2}$ near the endosymbiont from the enzyme carbonic anhydrase (CA) located around the symbiosome, fostering the algae photosynthetic process. Another example is in the nitrogen metabolism, which is necessary to the algae in the form of ammonium $\left(\mathrm{NH}_{3}^{+}\right)$and nitrate $\left(\mathrm{NO}_{3}^{-}\right)$, to form amino acids, and which are also a resource to the coral because they are transported back to the host as a nutrient. Calcification also is promoted as a consequence of the metabolic coupling of both organisms, since dissolved inorganic carbon necessary for photosynthesis by Symbiodinium is also necessary to produce calcium skeleton, which is mainly composed of calcium carbonate $\left(\mathrm{CaCO}_{3}\right)$ crystallized in an organic matrix. Calcification is enhanced by light in presence of Symbiodinium, and this affects the balance of dissolved inorganic carbon, taking $\mathrm{CO}_{2}$ for photosynthesis reactions, which directly promotes the precipitation of
$\mathrm{CaCO}_{3}$. In addition, organic products of photosynthesis are precursors of the synthesis of organic matrix, and energy and oxygen supplementation to the host increases its metabolism and promotes faster calcification $[33,45]$. Moreover, corals presenting Symbiodinium are capable of living in clear and shallow waters, where they are constantly threatened by high intensity solar radiation. Despite the fact that light levels are harmful, symbionts produce UV-absorbing sunscreen compounds, like mycosporine amino acids (MAAs), which act as light-harvesting pigments and free radical scavengers [46].

In summary, there are both metabolic coupling and regulation between Symbiodinium and host cells. The latter promotes their growth, reproduction, and survival at different organizational levels. We organize the levels of interaction of the endosymbiotic interaction as follows (see Table 1):

(1) Biochemical traffic level: related to the biochemical relationship and nutrient traffic inside the cells of the coral, and between the coral and Symbiodinium cells. 
TABLE 1: Representing ecological interactions using reaction networks.

\begin{tabular}{lcccc}
\hline Interaction & Positive & Neutral & Negative & Reaction \\
\hline Amensalism & - & $x$ & $y$ & $x+y \longrightarrow x$ \\
Antagonism & $x$ & - & $y$ & $x+y \longrightarrow 2 x$ \\
Mutualism & $x, y$ & - & - & $x+y \longrightarrow 2 x+2 y$ \\
Commensalism & $y$ & - & $x, y$ & $x+y \longrightarrow x+2 y$ \\
Competition & $x, y$ & $x+y \longrightarrow x$ and $x+y \longrightarrow y$ \\
\hline
\end{tabular}

(2) Cellular proliferation level: related to the cellular reproduction to achieve the dynamic stability state between the cells biomass of both partners.

(3) Organismic level: related to the growth of the coral and the protection from biological and mechanical erosion due to the calcidodermis production generated in lower levels.

Therefore, when the endosymbiotic interaction is established, the above explained processes at these three levels intertwine benefiting the persistence of endosymbiosis.

\section{Reaction Network Modeling of Ecological Interactions}

A reaction network is defined by a pair $(M, R)$, where $M=$ $\{a, b, c, \ldots\}$ is a set of molecular species, and $R \subseteq \mathscr{P}_{m}(M) \times$ $\mathscr{P}_{m}(M)$ is a set of reactions, where $\mathscr{P}_{m}(M)$ denotes the set of multisets of $M$. For example, consider

$$
\begin{aligned}
M & =\{a, b, c\}, \\
R & =\left\{r_{1}, r_{2}, r_{3}, r_{4}, r_{5}, r_{6}\right\}, \text { with, } \\
r_{1} & =a \longrightarrow 2 a, \\
r_{2} & =a+c \longrightarrow c, \\
r_{3} & =b+c \longrightarrow b+2 c, \\
r_{4} & =a \longrightarrow \varnothing, \\
r_{5} & =c \longrightarrow \varnothing, \\
r_{6} & =b+a \longrightarrow \varnothing .
\end{aligned}
$$

Note that reaction $r_{1}=a \longrightarrow 2 a$ represents a self-reproduction process of species $a$, reaction $r_{2}=a+c \longrightarrow c$ represents the destruction of species $a$ out of the interaction of species $a$ and $c$, and $r_{3}=b+c \longrightarrow b+2 c$ represents the reproduction of species $c$ catalyzed by $b$. Similarly, the destruction and disappearance of $a$ and $c$ are represented by $r_{4}$ and $r_{5}$, respectively, and the mutual destruction of $a$ and $b$ is represented by $r_{6}$.

The dynamics of a reaction network can be modeled using difference, stochastic, or differential equations [47], and a vast amount of literature is devoted to complement these approaches with structural analysis of the network [18-20].

Recently, reaction networks have been proposed to represent ecological interactions and ecosystems $[28,48]$. In particular, contrary to traditional network approaches, which represent different ecological interactions by different types of links, reaction networks characterize types of ecological interactions by the way in which combinations of inputs produce combinations of outputs. This opens up an exponentially wider range of interacting processes. Typical ecological interactions such as depredation, cooperation, and parasitism are easily expressed by means of reaction networks. For example, reaction $r_{3}=a+c \longrightarrow c$ of the reaction network equation (1) corresponds to an amensalistic interaction, since $a$ is destroyed in the presence of $c$ without altering $c$. Likewise, $r_{4}=b+c \longrightarrow b+2 c$ is a commensalistic relation, because $c$ benefits from its interaction with $b$ without altering $b$. In Table 1 , we represent ecological interactions using minimal reaction networks.

Interestingly, when one is interested in representing an ecological interaction in more detail, the model of the interaction is made by a collection of reactions representing the interaction mechanism. Since the interaction mechanisms of different interaction might share some species at both resource and product levels, an ecological system is modeled by a large reaction network composed by the coupling of multiple subnetworks, each of these subnetworks representing one of the ecological interactions of the ecosystem [28].

In addition to the structural transformational relation specified by a reaction, there is usually a parameter which indicates its likelihood to happen. This value, known as kinetic parameter or kinetic rate, is indicated above the arrow which specifies the reaction. For example, in the case of competition, the reactions $r_{1}=x+y \longrightarrow k_{x} x, r_{2}=x+$ $y \longrightarrow^{k_{y}} y$ constrained to $k_{x}<k_{y}$ would indicate that $y$ has a competitive advantage over $x$, as reaction $r_{2}$ is more likely to happen than $r_{1}$.

Moreover, the empty set symbol $\varnothing$ represents the environment, i.e., what is not part of the system under study. For this reason, reactions $\varnothing \longrightarrow^{k} a$ and $a \longrightarrow^{k} \varnothing$ indicate the inflow (born, entrance, spontaneous formation) of $a$ in the system while indicates the outflow (death, exit, decay) of $a$ from the system.

\section{Multilevel Reaction Network Model of Endosymbiotic Interactions}

In this section, we develop a reaction network model of the biochemical traffic and cellular proliferation levels of the endosymbiotic interaction depicted in Table 2. Next, we explain how to represent the regulation mechanisms occurring between these two levels. This model extends previous work [49].

4.1. Biochemical Traffic Level. At this level, endosymbiosis is characterized by the possibility to metabolize elements that coral cells alone cannot metabolize, and by the increase of 
TABLE 2: Levels of interaction and processes that are enhanced by endosymbiosis at each level.

\begin{tabular}{lr}
\hline Level of interaction & Processes driven by endosymbiosis \\
\hline Biochemical traffic & $\begin{array}{c}\text { Biochemical interrelationship, nutrient traffic, energy flow, } \\
\text { and calcidodermis biochemical production. }\end{array}$ \\
Cellular proliferation & Coral growth and protection against predators and other physical threats \\
\hline
\end{tabular}

calcium production, which leads to a macroscopic calcium layer that protects the macroscopic coral from predators and erosion. The following reaction network represents in a simplified way the endosymbiotic interaction at the biochemical traffic level:

Species set:

$R_{H}$ : host cell resource

\author{
$E$ : energy \\ $R_{C}$ : resource to form calcium layer \\ $\overline{R_{C}}$ : resource that is product of the calcium formation \\ reaction \\ $\mathrm{Cal}_{C}:$ calcium
}

The reactions of a cell without Symbiodinium in it are

$$
\begin{aligned}
& r_{t_{1}}=\varnothing \stackrel{k_{1}}{\longrightarrow} R_{H}: \text { inflow of resource for the host cell, } \\
& r_{t_{2}}=\varnothing \stackrel{k_{2}}{\longrightarrow} R_{C} \text { : inflow of resource for calcium formation, } \\
& r_{t_{3}}=\varnothing \stackrel{k_{3}}{\longrightarrow} \overline{R_{C}} \text { : inflow of resource for Symbiodinium (not useful without endosymbiosis), } \\
& r_{t_{4}}=R_{H} \stackrel{k_{4}}{\longrightarrow} E \text { : energy generation by host cell, } \\
& r_{t_{5}}=E+R_{C} \stackrel{k_{5}}{\longrightarrow} \overline{R_{C}}+\text { Cal : calcium formation reaction, } \\
& r_{t_{6}}=E \stackrel{k_{\text {Eloss }}}{\longrightarrow} \varnothing \text { : energy consumption. }
\end{aligned}
$$

Note that in the model given by equation (2) there is an energy generation pathway given by the reaction sequence $\left(r_{t_{1}}, r_{t_{4}}\right)$, which in turns activate the calcium formation pathway $\left(r_{t_{2}}, r_{t_{5}}\right)$. For simplicity, the energy consumption pathway is represented by $r_{t_{6}}$. Note that if $k_{1}$ is too small, meaning that there is not enough $R_{H}$ available in the environment, the energy generation process driven by $r_{t_{4}}$ might not compensate for the energy consumption represented by $r_{t_{6}}$.

When the Symbiodinium is present in the cell, we add the following reaction to the model:

$$
r_{t_{7}}=\overline{R_{C}} \stackrel{k_{7}}{\longrightarrow} E+x \text { : energy and by - product generation by Symbiodinium, }
$$

meaning that Symbiodinium is capable of generating energy for the cell from the resource $\overline{R_{C}}$ and creating $x$, which correspond to photosynthetic byproducts of algae metabolism, that can be sensed by the host. This last reaction activates a new energy generation pathway $\left(r_{t_{3}}, r_{t_{7}}\right)$ by using up the byproduct $\overline{R_{C}}$ of $r_{t_{5}}$, and hence metabolizing $\bar{R}_{C}$ to produce more $\mathrm{Cal}$. The latter modifies the chemical equilibrium between $R_{C}$ and $\bar{R}_{C}$ by taking up more $\bar{R}_{C}$ for its photosynthetic metabolism, which directly favors the precipitation of Cal by Le Chatelier's principle [33]. 
4.2. Cellular Proliferation Level. At the cellular proliferation level, we identify three types of cells:

\section{$H$ : host}

\section{S: Symbiodinium}

HS: symbiont (host and Symbiodinium interacting in endosymbiosis)
Similar to the previous case, we will keep the model very simple, so we introduce reactions that account for the most basic interactions between cells, cell proliferation, and endosymbiosis. Other aspects such as the multiple manners in which the cells can die, or the environmental conditions that foster their reproduction, are not included.

$$
\begin{aligned}
& r_{p_{1}}=H \stackrel{k_{H}^{\text {rep }}}{\longrightarrow} 2 H: \text { (H reproduces), } \\
& \left.r_{p_{2}}=S \stackrel{k_{S}^{\text {rep }}}{\longrightarrow} 2 S: \text { ( } S \text { reproduces }\right), \\
& r_{p_{3}}=H+S \stackrel{k_{H S}^{\text {form }} \longrightarrow}{\longrightarrow} \text { HS: (HS is formed), } \\
& r_{p_{4}}=H S \stackrel{k_{H S}^{\text {rep }}}{\longrightarrow} 2 H S: \text { (HS reproduces), } \\
& r_{p_{5}}=H \stackrel{k_{H}^{\mathrm{die}}}{\longrightarrow} \varnothing:(H \text { dies }), \\
& r_{p_{6}}=S \stackrel{k_{S}^{\text {die }}}{\longrightarrow} \varnothing:(S \text { dies }), \\
& r_{p_{7}}=H S \stackrel{k_{H S}^{\text {die }}}{\longrightarrow} \varnothing:(H S \text { dies }) .
\end{aligned}
$$

Under appropriate internal cell proliferation conditions $k_{\mathrm{HS}}^{\text {rep }}>k_{S}^{\text {rep }}$, and $k_{\mathrm{HS}}^{\text {rep }}>k_{H}^{\text {rep }}$. This is because the Symbiodinium is supposed to have more energy due to its more efficient metabolic activity. Similarly, $k_{\mathrm{HS}}^{\text {die }}<k_{S}^{\text {die }}, k_{\mathrm{HS}}^{\text {die }}<k_{H}^{\text {die because HS }}$ has a larger calcium layer, which protects it best from predators, and because HS has a more diverse metabolism. On the other hand, under cell proliferation conditions that become harmful to the coral we expect $k_{\mathrm{HS}}^{\text {rep }}<k_{S}^{\text {rep }}$, and $k_{\mathrm{HS}}^{\text {rep }}<k_{H}^{\text {rep }}$ because the organism releases inhibitory factors that reduce its tendency to reproduce, and $k_{\mathrm{HS}}^{\text {die }}>k_{S}^{\text {die }}$, and $k_{\mathrm{HS}}^{\text {die }}>k_{H}^{\text {die }}$ due to Symbiodinium cell expulsion or degradation of HS.

4.3. Levels of Association and Regulation: An Example. As seen in Section 2, it is possible to identify variables in one level that constrain or promote the activity at other levels. In this model, we consider only one top-down constraint, and one bottom-up promoter, by modifying the kinetic parameter $k_{\mathrm{HS}}^{\text {rep }}$ as an example of how to represent the multilevel control mechanisms.

For the bottom-up control mechanism, we have that the nutrient traffic level reactions $r_{t_{4}}$ and $r_{t_{7}}$ produce energy, and that such energy is consumed by reactions $r_{t_{5}}$ and $r_{t_{6}}$ for metabolic survival. Therefore, when the production of energy is not enough to compensate its consumption, the cell HS will not reproduce, so $r_{p_{4}}$ becomes inactive. Analogously, when the production of energy surpasses its consumption, $r_{p_{4}}$ becomes active. Hence, we consider a threshold of total energy production in the lower level $E^{*}$, and when the energy in the nutrient traffic level surpasses this threshold, the kinetic parameter $k_{\mathrm{HS}}^{\text {rep }}$ activates.

For the top-down direction, we consider a signaling species that is known to regulate the proliferation of the Symbiodinium according to its sensitivity to some photosynthetic byproducts. The photosynthetic product is in this case represented by the species $x$ at the nutrient traffic level (see $r_{t_{7}}$ in equation (2)). Let the signaling species be $F_{\text {inh }}$, representing the factor that is released when the endosymbiont's byproducts $x$ surpass a threshold $x^{*}$, and that is sensed by the host, deactivating the reaction $r_{t_{7}}$.

In order to provide a formal specification of the bottomup and top-down regulatory mechanism of HS reproduction, we introduce the following reactions at the organismic level:

$$
\begin{aligned}
& r_{p_{8}}=\mathrm{HS} \stackrel{k_{p_{8}}}{\longrightarrow} \mathrm{HS}+F_{\mathrm{inh}}: \text { inhibition factor released by the host }, \\
& r_{p_{9}}=F_{\mathrm{inh}} \stackrel{k_{p_{9}}}{\longrightarrow} \varnothing: \text { natural degradation of the inhibition factor, }
\end{aligned}
$$

where $k_{p_{8}}=h\left(x-x^{*}\right)$, with $h\left(x-x^{*}\right)$ being the known Heaviside-step function, which is zero if $x-x^{*} \leq 0$ and a reference value (one for simplicity) if $x-x^{*}>0$. Hence, reaction $r_{p_{8}}$ is inactive when the concentration of $x$ is below the threshold $x^{*}$ and once the concentration $x$ surpasses the threshold $x^{*}$ the inhibition factor is released, 


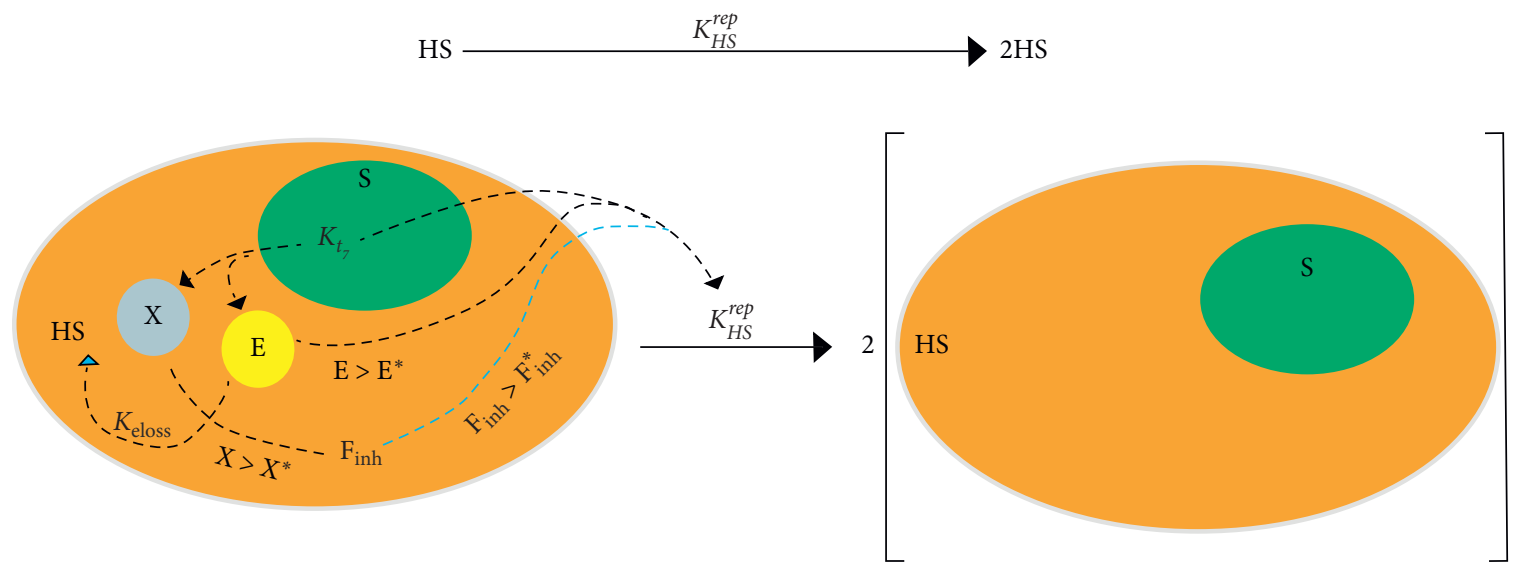

(a)

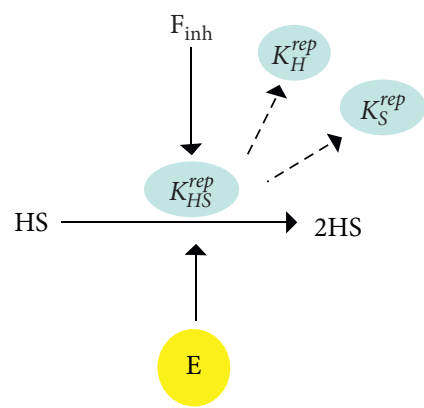

(b)
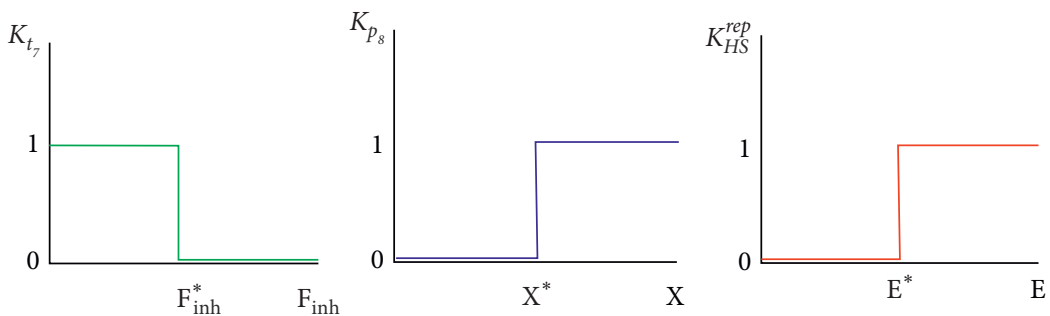

$K_{t}$

$K_{p_{s}}$

$K_{H S}^{r e p}$

(c)

Figure 3: Schema of the regulation of species through two levels of interaction. (a) Representation of the interaction between the host $(H$ in orange) and the Symbiodinium ( $S$ in green) in the reaction $r_{p_{4}}$, and how $E, x$, and $F_{\text {inh }}$ affect the kinetic parameter $k_{\mathrm{HS}}^{\text {rep }}$. Dotted lines represent ways of regulation of $k_{\mathrm{HS}}^{\text {rep }}$, occurring when $E>E^{*}, x>x^{*}$, and $F_{\mathrm{inh}}>F_{\text {inh }}^{*}$. Blue dotted line represents the release of inhibition factors by coral cells. (b) Regulation of $k_{\mathrm{HS}}^{\text {rep }}$, by $F_{\mathrm{inh}}$ and $E$, showing the indirect regulation of $k_{S}^{\text {rep }}$ and $k_{H}^{\text {rep }}$ in dotted lines. (c) Dependency of $k_{t_{7}}, k_{p_{8}}$, and $k_{\mathrm{HS}}^{\text {rep }}$ on $F_{\text {inh }}, x$, and $E$, respectively.

while $k_{p_{9}}$ is a constant rate controlling the degradation of $F_{\text {inh. }}$.

Next, the kinetic parameters $k_{t_{7}}$ becomes explicitly dependent on $F_{\text {inh }}$ to control the extra production of energy and $x$ :

$$
k_{t_{7}}=h\left(F_{\text {inh }}-F_{\text {inh }}^{*}\right),
$$

where $F_{\text {inh }}^{*}$ is the $F_{\text {inh }}$ tolerance threshold that once surpassed the production of extra energy is inhibited.

Finally, we modify $k_{\mathrm{HS}}^{\text {rep }}$, making it dependent on $E$ as follows:

$$
k_{\mathrm{HS}}^{\text {rep }}=h\left(E-E^{*}\right) .
$$

Therefore, when the nutrient traffic is intensified by the presence of the Symbiodinium, the extra energy produced regulates in a bottom-up manner the kinetic parameter $k_{\mathrm{HS}}^{\mathrm{rep}}$ of the endosymbiotic interaction at the cellular proliferation level, activating its proliferation. Conversely, when proliferation of Symbiodinium surpasses the amount of the byproducts required for operating its metabolism, $F_{\text {inh }}$ is released, inhibiting in a topdown manner the kinetic parameter $k_{t_{7}}$. We represent 


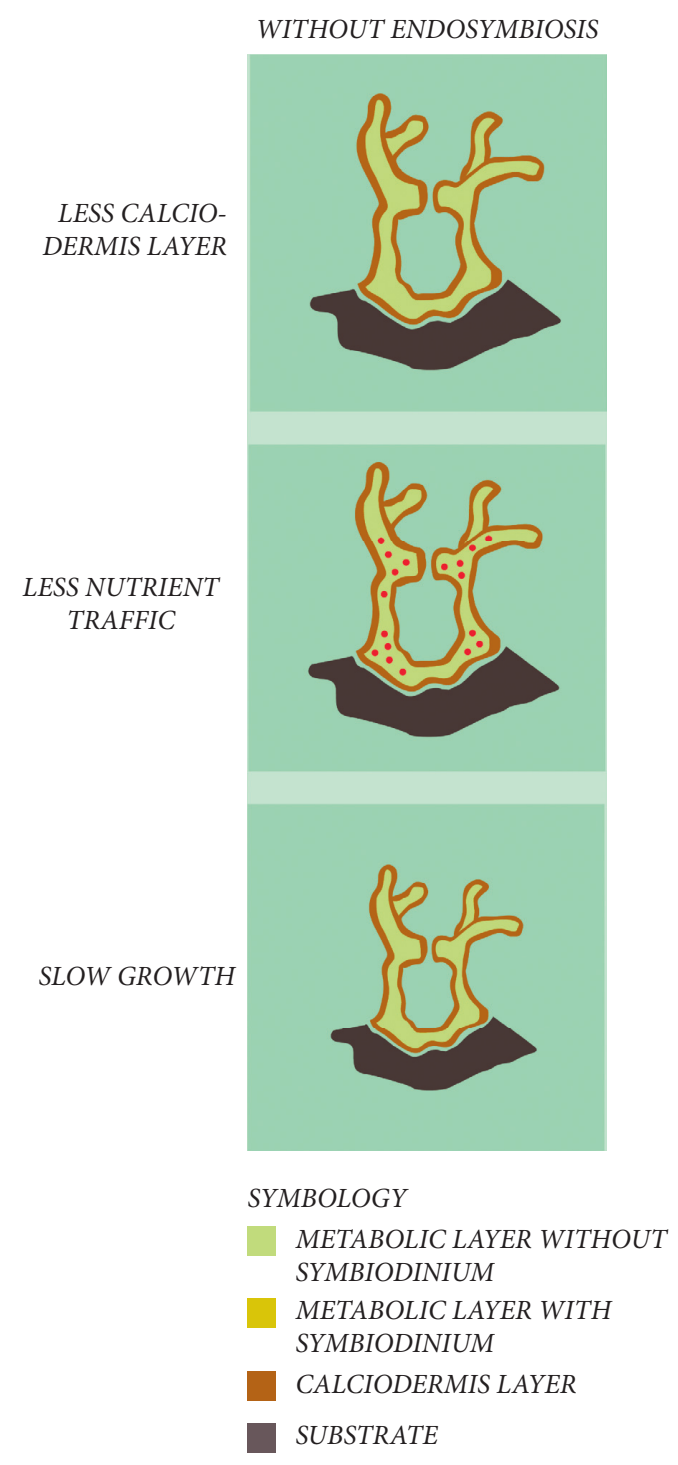

(a)

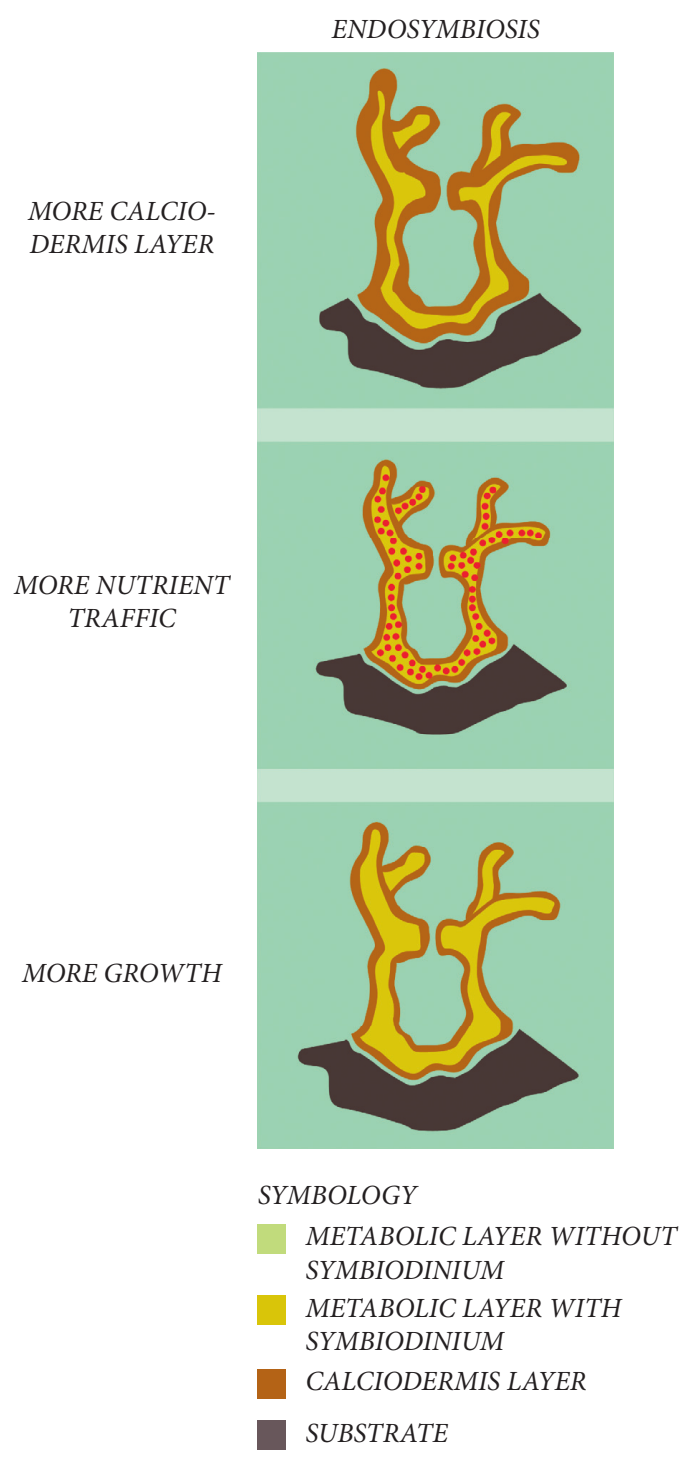

(b)

Figure 4: Organismal system processes, showing in a comparative scenario how different processes as calcidodermis production, nutrient traffic, and coral growth are enhanced by the endosymbiosis interaction (b) with respect to the coral without Symbiodinium (a).

these interrelationships in Figure 3, specifically the regulation for $k_{\mathrm{HS}}^{\text {rep }}$.

\section{Discussion}

A key concept in biological complexity is the emergence of properties that coregulate the persistence of processes occurring at different levels, and which are needed for organisms to live and reproduce. In this sense, the representation of biological phenomena as a hierarchy of intertwined dynamical processes is an important challenge to advance our understanding of biological complexity.

Coral endosymbiosis presents an interesting opportunity to study this issue. First, there is a concrete evidence of the microscopic interaction between the Symbiodinium and the coral host cell as well as evidence of the micro- and macroscopic consequences of such interaction. Secondly, levels of interaction can be clearly delineated. Namely, in our model we considered a nutrient traffic and cellular proliferation levels, where the former is undoubtedly nested in the other. Thirdly, as explained in Section 4.3, even in our simple two-levels model, bottom-up and top-down regulatory mechanisms occur. Therefore, the coral-Symbiodinium endosymbiotic interaction exhibits structural features allowing a simple but explicative model of its mechanism.

Reaction network-like models of biological interactions have only recently been investigated $[28,48,49]$, but seem to provide the necessary versatility to incorporate multilevel regulatory mechanisms in a sensible way. However, this approach is in its infancy and requires extensions.

We propose two ways in which our model can be extended. The first is to give a more detailed account of what occurs at each level, and the second is to incorporate more levels. For the former case, a more detailed account of the 
reproduction of the symbionts within the endosymbiont could be developed. For example, we can introduce a new species $\mathrm{HS}_{2}$ meaning that the host has two endosymbionts in it, and incorporate the reactions $\mathrm{HS} \longrightarrow \mathrm{HS}_{2}$ and $\mathrm{HS}_{2} \longrightarrow 2 \mathrm{HS}$ to represent the replication process of the endosymbiont. Analogously, it is possible to incorporate other necessary steps to provide a more realistic account of the phenomena such as regulation at the recognition level [50], symbiosome maturation [51], and regulation of symbiont biomass by symbiont expulsion and degradation $[32,52]$. For the latter case, we can incorporate the macroscopic organismic level in our model, involving the coral's growth and survival. The simplest model of the endosymbiotic organismic activity lies at comparing the coral's ability to grow, with and without Symbiodinium (named $S$ and $C$, respectively) under mechanical and biological erosion (Er), and its ability to reproduce. The coral's ability to grow is directly related to nutrient traffic and calcidodermis strengthening as depicted in Figure 4. For simplicity, we will provide an example focusing on the growth of $C$, in the presence of $S$, and erosion Er. Other aspects such as the reproduction of erosion factors (e.g., fish that predates the coral), or natural death of the $S$ and $C$, are not incorporated.

$$
\begin{aligned}
& r_{o_{1}}=S+\operatorname{Er} \stackrel{k_{S}^{\text {dep }}}{\longrightarrow} \text { Er: }(\text { Er destroys } S), \\
& r_{o_{2}}=C+\operatorname{Er} \stackrel{k_{C}^{\text {dep }}}{\longrightarrow} \text { Er: }(\text { Er destroys } C), \\
& r_{o_{3}}=S \stackrel{k_{S}^{\text {rep }}}{\longrightarrow} 2 S:(S \text { growth }), \\
& r_{o_{4}}=C \stackrel{k_{C}^{\text {rep }}}{\longrightarrow} 2 C:(C \text { growth }) .
\end{aligned}
$$

The values $k_{S}^{\text {dep }}, k_{C}^{\text {dep }}$ and $k_{S}^{\text {rep }}, k_{C}^{\text {rep }}$ are the mechanical and biological erosion and growth rates of $S$ and $C$, respectively.

Note that in this case the interactions that $C$ and $S$ have with the other species are equal. Therefore, the parameters $k_{S}^{\text {dep }}, k_{C}^{\text {dep }}$ modulate their susceptibility to be destroyed by environmental phenomena or by predation, while $k_{S}^{\text {rep }}, k_{C}^{\text {rep }}$ modulate their growth rate. For a more precise model of the general organismal situation, it is necessary to relate the organismal level to parameters of the lower levels such as the rate of production of the calcium layer $\left(r_{t_{5}}\right)$, the free internal metabolic energy (related to the increase of the biochemical traffic), and the rate of proliferation of the cells.

On a more technical note, future models should apply reaction networks nested in compartments $[53,54]$ instead of traditional reaction networks. Such models should be more realistic, but also grow in size and complexity. Thus, it will become necessary to apply pathway analysis methods such as flux-balance analysis [55] and chemical organization theory [19] to identify dynamical features that can be linked to the properties of the interactions, and to study how structural perturbations to the system such as the addition of new biochemical components, or new macroscopic species, affect the stability landscape of the system.

\section{Conclusion}

The complexity of biological processes is related to the integration of the different levels at which they occur and to the emergent properties that characterize the gaps between such levels. To address this, it is necessary to model not only the different levels at which interactions manifest, but also the interaction between these levels through multilevel regulation mechanisms. We developed a simple mechanistic model for the endosymbiotic interaction between corals and the algae (Symbiodinium) using reaction networks.

In our model, which complements a reaction network model of endosymbiosis between corals and dinoflagellates of the genus Symbiodinium [49], we incorporate biochemical species $\left(R_{H}, R_{C}, \bar{R}_{C}, \mathrm{Cal}\right.$, and $\left.x\right)$, thermodynamic species $(E)$, cellular species $(H, S$, and $\mathrm{HS})$, and a regulatory factor $\left(F_{\text {inh }}\right)$. In addition, we incorporated a bilevel regulatory mechanism for the proliferation of the endosymbiotic cell HS which is fostered by the increase of its energy (occurring at the nutrient traffic level) but also regulated by the release of $F_{\text {inh }}$ when the byproduct $x$ of the energy production surpasses a threshold (occurring at the cellular proliferation level). This mechanism promotes the controlled organism's reproduction and is coherent with the knowledge of this interaction $[11,56]$.

The idea of developing multilevel interaction models for ecological interactions is relatively new in theoretical biology, as there are not many works that modulate kinetic parameters from the concentration of the system species at different levels [17]. The major innovation of this work is to consider that kinetic parameters at one level can be modulated by the presence of species (biotic or abiotic) at other levels, and thus providing a first approach to generate an integrative view of the multilevel nature of the endosymbiotic relationship.

As a final remark, we consider that this approach has the potential to describe the complexity of not only endosymbiosis, but also other complex multilevel biological interactions such as physiological approach in multiscale system biology [57, 58], cancer biology [59], and multiscale ecological approaches $[60,61]$.

\section{Data Availability}

Our study is theoretical. Therefore, no data were used to support this study.

\section{Conflicts of Interest}

The authors declare that they have no conflicts of interest.

\section{Acknowledgments}

The first author was funded by the John Templeton Foundation "The Origins of Goal-Directedness: A Formal Scenario Based on Chemical Organization Theory and Cybernetics" under Grant ID\# 61733. The second author was funded by ANID-PFCHA/Doctorado Nacional/2019 under Grant no. 21191885. 


\section{References}

[1] U. Kutschera and K. J. Niklas, "Endosymbiosis, cell evolution, and speciation," Theory in Biosciences, vol. 124, no. 1, pp. 1-24, 2005.

[2] D. M. Wilkinson, "At cross purposes," Nature, vol. 412, no. 6846, p. $485,2001$.

[3] J. M. Archibald, "Endosymbiosis and eukaryotic cell evolution," Current Biology, vol. 25, no. 19, pp. R911-R921, 2015.

[4] J. J. Wernegreen, "Endosymbiosis: lessons in conflict resolution," PLoS Biology, vol. 2, no. 3, p. e68, 2004.

[5] C. Darwin and J. W. Burrow, "The origin of species," 1859.

[6] R. Rowan, "Diversity and ecology of zooxanthellae on coral reefs," Journal of Phycology, vol. 34, no. 3, pp. 407-417, 1998.

[7] A. C. Baker, "Symbiont diversity on coral reefs and its relationship to bleaching resistance and resilience," Coral Health and Disease. In Coral Health and Disease, vol. 1, pp. 177-194, 2004.

[8] D. Yellowlees, T. A. V. Rees, and W. Leggat, "Metabolic interactions between algal symbionts and invertebrate hosts," Plant, Cell \& Environment, vol. 31, no. 5, pp. 679-694, 2008.

[9] P. J. McAuley, "The cell cycle of symbiotic Chlorella. I. the relationship between host feeding and algal cell growth and division," Journal of Cell Science, vol. 77, no. 1, pp. 225-239, 1985.

[10] P. J. McAuley, "Temporal relationships of host cell and algal mitosis in the green hydra symbiosis," Journal of Cell Science, vol. 58, no. 1, pp. 423-431, 1982.

[11] P. L. Antonelli, S. F. Rutz, P. W. Sammarco, and K. B. Strychar, "Evolution of symbiosis in hermatypic corals: a model of the past, present, and future," Nonlinear Analysis: Real World Applications, vol. 32, pp. 389-402, 2016.

[12] P. Beldade, K. Koops, and P. M. Brakefield, "Developmental constraints versus flexibility in morphological evolution," Nature, vol. 416, no. 6883, pp. 844-847, 2002.

[13] J. Sánchez-Hernández, F. Cobo, and P. A. Amundsen, "Food web topology in high mountain lakes," PLoS One, vol. 10, no. 11, Article ID e0143016, 2015.

[14] S. J. Arnold, "Constraints on phenotypic evolution," The American Naturalist, vol. 140, pp. S85-S107, 1992.

[15] D. T. Fraebel, H. Mickalide, D. Schnitkey, J. Merritt, T. E. Kuhlman, and S. Kuehn, "Environment determines evolutionary trajectory in a constrained phenotypic space," Elife, vol. 6, Article ID e24669, 2017.

[16] V. N. Reddy, M. L. Mavrovouniotis, and M. N. Liebman, "Petri net representations in metabolic pathways," ISMBIndian Standard Medium Weight Beam, vol. 93, pp. 328-336, 1993.

[17] R. Bardini, G. Politano, A. Benso, and S. Di Carlo, "Multi-level and hybrid modelling approaches for systems biology," Computational and Structural Biotechnology Journal, vol. 15, pp. 396-402, 2017.

[18] D. J. Wilkinson, Stochastic Modelling for Systems Biology, CRC Press, Boca Raton, FL, USA, 2011.

[19] P. Dittrich and P. S. di Fenizio, "Chemical organisation theory," Bulletin of Mathematical Biology, vol. 69, no. 4, pp. 1199-1231, 2007.

[20] D. A. Fell, "Metabolic control analysis: a survey of its theoretical and experimental development," Biochemical Journal, vol. 286, no. 2, p. 313, 1992.

[21] M. Feinberg and F. J. M. Horn, "Dynamics of open chemical systems and the algebraic structure of the underlying reaction network," Chemical Engineering Science, vol. 29, no. 3, pp. 775-787, 1974.
[22] P. Dittrich and L. Winter, "Reaction networks as a formal mechanism to explain social phenomena," in Proceedings of the Fourth International Workshop on Agent-Based Approaches in Economics and Social Complex Systems, pp. 9-13, New York, NY, USA, May 2005.

[23] P. Dittrich and L. Winter, "Chemical organizations in a toy model of the political system," Advances in Complex Systems, vol. 11, no. 4, pp. 609-627, 2008.

[24] T. Veloz, P. Razeto-Barry, P. Dittrich, and A. Fajardo, "Reaction networks and evolutionary game theory," Journal of Mathematical Biology, vol. 68, no. 1-2, pp. 181-206, 2014

[25] D. Velegol, P. Suhey, J. Connolly, N. Morrissey, and L. Cook, "Chemical game theory," Industrial \& Engineering Chemistry Research, vol. 57, no. 41, pp. 13593-13607, 2018.

[26] T. Veloz, "Teoría de organizaciones químicas: un lenguaje formal para la autopoiesis y el medio ambiente," in Un Concepto Vivo, P. Razeto-Barry and R. Ramos-Jiliberto, Eds., pp. 229-245, Editorial Nueva Civilización, Santiago, Chile, 2013.

[27] T. Veloz and P. Razeto-Barry, "Reaction networks as a language for systemic modeling: fundamentals and examples," Systems, vol. 5, no. 1, p. 11, 2017.

[28] T. Veloz, "The complexity-stability debate, chemical organization theory, and the identification of non-classical structures in ecology," Foundations of Science, vol. 25, no. 1, pp. 259-273, 2019.

[29] P. S. Davies, "Effect of daylight variations on the energy budgets of shallow-water corals," Marine Biology, vol. 108, no. 1, pp. 137-144, 1991.

[30] S. K. Davy, D. Allemand, and V. M. Weis, "Cell biology of cnidarian-dinoflagellate symbiosis," Microbiology and Molecular Biology Reviews, vol. 76, no. 2, pp. 229-261, 2012.

[31] J. E. Loram, H. G. Trapido-Rosenthal, and A. E. Douglas, "Functional significance of genetically different symbiotic algae Symbiodinium in a coral reef symbiosis," Molecular Ecology, vol. 16, no. 22, pp. 4849-4857, 2007.

[32] W. Fitt, "Cellular growth of host and symbiont in a cnidarianzooxanthellar symbiosis," The Biological Bulletin, vol. 198, no. 1, pp. 110-120, 2000.

[33] S. Tambutté, M. Holcomb, C. Ferrier-Pagès et al., "Coral biomineralization: from the gene to the environment," Journal of Experimental Marine Biology and Ecology, vol. 408, no. 1-2, pp. 58-78, 2011.

[34] J.-P. Gattuso, D. Allemand, and M. Frankignoulle, "Photosynthesis and calcification at cellular, organismal and community levels in coral reefs: a review on interactions and control by carbonate chemistry," American Zoologist, vol. 39, no. 1, pp. 160-183, 1999.

[35] V. M. Weis, "Cellular mechanisms of Cnidarian bleaching: stress causes the collapse of symbiosis," Journal of Experimental Biology, vol. 211, no. 19, pp. 3059-3066, 2008.

[36] T. P. Hughes, J. T. Kerry, M. Álvarez-Noriega et al., "Global warming and recurrent mass bleaching of corals," Nature, vol. 543, no. 7645, pp. 373-377, 2017.

[37] N. Neckelmann and L. Muscatine, "Regulatory mechanisms maintaining the Hydra-Chlorella symbiosis," Proceedings of the Royal society of London. Series B. Biological sciences, vol. 219, no. 1215, pp. 193-210, 1983.

[38] C. E. Taylor, L. Muscatine, and D. R. Jefferson, "Maintenance and breakdown of the Hydra-Chlorella symbiosis: a computer model," Proceedings of the Royal Society of London B Biological Sciences, vol. 238, no. 1292, pp. 277-289, 1989.

[39] R. D. Gates and L. Muscatine, "Three methods for isolating viable anthozoan endoderm cells with their intracellular 
symbiotic dinoflagellates," Coral Reefs, vol. 11, no. 3, pp. 143-145, 1992.

[40] L. Muscatine, C. Ferrier-Pages, A. Blackburn, R. D. Gates, G. Baghdasarian, and D. Allemand, "Cell-specific density of symbiotic dinoflagellates in tropical anthozoans," Coral Reefs, vol. 17, no. 4, pp. 329-337, 1998.

[41] A. Douglas and D. C. Smith, "The green hydra symbiosis. VIII. mechanisms in symbiont regulation," Proceedings of the Royal Society of London. Series B. Biological Sciences, vol. 221, no. 1224, pp. 291-319, 1984.

[42] P. G. Falkowski, Z. Dubinsky, L. Muscatine, and L. McCloskey, "Population control in symbiotic corals," BioScience, vol. 43, no. 9, pp. 606-611, 1993.

[43] G. J. Smith and L. Muscatine, "Cell cycle of symbiotic dinoflagellates: variation in G1 phase-duration with anemone nutritional status and macronutrient supply in the Aiptasia pulchella-Symbiodinium pulchrorum symbiosis," Marine Biology, vol. 134, no. 3, pp. 405-418, 1999.

[44] B. R. Gordon and W. Leggat, "Symbiodinium-Invertebrate symbioses and the role of metabolomics," Marine Drugs, vol. 8, no. 10, pp. 2546-2568, 2010.

[45] D. Allemand, E. Tambutté, D. Zoccola, and S. Tambutté, "Coral calcification, cells to reefs," Coral Reefs: An Ecosystem in Transition, vol. 99, pp. 119-150, 2011.

[46] N. N. Rosic, "Phylogenetic analysis of genes involved in mycosporine-like amino acid biosynthesis in symbiotic dinoflagellates," Applied Microbiology and Biotechnology, vol. 94, no. 1, pp. 29-37, 2012.

[47] D. Angeli, "A tutorial on chemical reaction network dynamics," European Journal of Control, vol. 15, no. 3, pp. 398-406, 2009.

[48] C. Gaucherel and F. Pommereau, "Using discrete systems to exhaustively characterize the dynamics of an integrated ecosystem," Methods in Ecology and Evolution, vol. 10, no. 9, pp. 1615-1627, 2019.

[49] T. Veloz and D. Flores, "Towards endosymbiosis modeling using reaction networks," Soft Computing, vol. 25, pp. 68316840, 2019.

[50] A. C. Baker, "Flexibility and specificity in coral-algal symbiosis: diversity, ecology, and biogeography of symbiodinium," Annual Review of Ecology, Evolution, and Systematics, vol. 34, no. 1, pp. 661-689, 2003.

[51] M.-C. Chen, M.-C. Hong, Y.-S. Huang, M.-C. Liu, Y.-M. Cheng, and L.-S. Fang, "ApRab11, a cnidarian homologue of the recycling regulatory protein Rab11, is involved in the establishment and maintenance of the Aiptasia-Symbiodinium endosymbiosis," Biochemical and biophysical research communications, vol. 338, no. 3, pp. 1607-1616, 2005.

[52] E. A. Titlyanov, T. V. Titlyanova, V. A. Leletkin, J. Tsukahara, R. Van Woesik, and K. Yamazato, "Degradation of zooxanthellae and regulation of their density in hermatypic corals," Marine Ecology Progress Series, vol. 139, pp. 167-178, 1996.

[53] H. Fellermann and L. Cardelli, "Programming chemistry in DNA-addressable bioreactors," Journal of The Royal Society Interface, vol. 11, no. 99, 2014.

[54] S. Peter and P. Dittrich, "On the relation between organizations and limit sets in chemical reaction systems," Advances in Complex Systems, vol. 14, pp. 77-96, 2011.

[55] J. D. Orth, I. Thiele, and B. ø. Palsson, "What is flux balance analysis?" Nature Biotechnology, vol. 28, no. 3, pp. 245-248, 2010.

[56] R. Cunning, E. B. Muller, R. D. Gates, and R. M. Nisbet, "A dynamic bioenergetic model for coral- Symbiodinium symbioses and coral bleaching as an alternate stable state," Journal of Theoretical Biology, vol. 431, pp. 49-62, 2017.

[57] J. O. Dada and P. Mendes, "Multi-scale modelling and simulation in systems biology," Integrative Biology, vol. 3, no. 2, pp. 86-96, 2011.

[58] H.-G. Holzhütter, D. Drasdo, T. Preusser, J. Lippert, and A. M. Henney, "The virtual liver: a multidisciplinary, multilevel challenge for systems biology," Wiley Interdisciplinary Reviews: Systems Biology and Medicine, vol. 4, no. 3, pp. 221-235, 2012.

[59] T. S. Deisboeck, Z. Wang, P. Macklin, and V. Cristini, "Multiscale cancer modeling," Annual Review of Biomedical Engineering, vol. 13, no. 1, pp. 127-155, 2011.

[60] N. Mayon, A. Bertrand, D. Leroy et al., "Multiscale approach of fish responses to different types of environmental contaminations: a case study," The Science of the Total Environment, vol. 367, no. 2-3, pp. 715-731, 2006.

[61] S. Rubin, T. Veloz, and P. Maldonado, "Beyond planetaryscale feedback self-regulation: gaia as an autopoietic system," Biosystems, vol. 199, Article ID 104314, 2021. 